\title{
SENSORES ELETROQUÍMICOS: CONSIDERAÇÕES SOBRE MECANISMOS DE FUNCIONAMENTO E APLICAÇÕES NO MONITORAMENTO DE ESPÉCIES QUÍMICAS EM AMBIENTES MICROSCÓPICOS
}

\author{
Denise Lowinsohn e Mauro Bertotti* \\ Instituto de Química - Universidade de São Paulo, CP 26077, 05513-970 São Paulo - SP, Brasil
}

Recebido em 28/6/05; aceito em 18/1/06; publicado na web em 11/8/06

\begin{abstract}
ELECTROCHEMICAL SENSORS: FUNDAMENTALS AND APPLICATIONS IN MICROENVIRONMENTS. Electrochemical sensors have attracted considerable attention in recent years because they provide data about the chemical state of our surroundings and the dynamics of the chemical transformations in the form a spatially resolved image. Particular interest has been directed to measurements in restricted-volume samples as new technologies enable the fabrication of miniaturized versions of sensors with reproducible characteristics. Taking these aspects into consideration, this review focuses on the use of electrodes of micrometer dimensions to acquire chemical information in microdomains in which concentrations may not be spatially homogeneous. This is possible because microelectrodes allow fast-response measurements with micrometer resolution to be performed. On the other hand, the use of microelectrodes as amperometric sensors presents an inherent drawback owing to the insufficient specificity toward the substrate of interest. Hence, some comments on strategies to enhance the selectivity of amperometric sensors are also made. Finally, recent applications of structurally microscopic electrodes as in vivo sensors are shown, as well as a prospect of the future trend in this field.
\end{abstract}

Keywords: electrochemical sensors; microelectrodes; in vivo measurements.

\section{INTRODUÇÃO}

\section{O atual panorama da Eletroanalítica}

As técnicas eletroquímicas constituem-se em poderosa ferramenta para os químicos analíticos na resolução de seus problemas, especialmente por causa de algumas características vantajosas como a elevada sensibilidade das determinações, custo moderado e portabilidade $^{1-3}$. A versatilidade das técnicas eletroquímicas também merece destaque visto que é possível controlar as reações eletródicas modificando a interface eletrodo-solução e selecionando-se criteriosamente o potencial aplicado à célula. A facilidade de automação em virtude da medição de sinais elétricos, a possibilidade de proceder à especiação de íons metálicos em certos casos e a "compatibilidade ambiental", pois o reagente empregado é o elétron, também consistem em aspectos que conferem aos métodos eletroanalíticos uma posição de destaque no contexto da Química Analítica.

Inerentes às propriedades acima destacadas, aparecem aspectos desvantajosos das medições eletroquímicas, principalmente quando comparadas às efetuadas com técnicas espectroscópicas. Um dos problemas refere-se à interação do eletrodo com a amostra, que em muitos casos ocasiona perda ou irreprodutibilidade nas medidas. $\mathrm{O}$ conjunto de compostos passíveis de determinação eletroanalítica também é menos abrangente que no caso das técnicas espectroscópicas. Finalmente, deve-se destacar a natural aversão às técnicas eletroquímicas em razão do sofisticado e nem sempre muito bem compreendido processo heterogêneo de transferência de elétrons que ocorre na interface eletrodo-solução.

No atual panorama da Eletroanalítica, pesquisas nas quais se utilizam detectores amperométricos acoplados a sistemas de separação têm recebido atenção crescente, especialmente nos casos em que se deseja a miniaturização do sistema analítico ${ }^{4-16}$. Uma justificativa para esta motivação inclui a disponibilidade de tecnologias que facilmente viabilizam a fabricação de eletrodos em escala

*e-mail: mbertott@iq.usp.br micrométrica, a preços relativamente baixos. Além disso, a sensibilidade das determinações não é comprometida pela redução do tamanho do sensor ou da célula, como é o caso dos métodos espectroscópicos, uma vez que a reação de interesse ocorre na região interfacial eletrodo/solução e, portanto, não depende do volume da amostra. Alta sensibilidade analítica pode também ser obtida empregando-se espectroscopia de massa ou fluorescência por laser induzido ${ }^{17}$. Entretanto, os instrumentos requeridos para o emprego destas técnicas são muito mais caros e de maior porte que um potenciostato, o qual pode ser facilmente construído em um "chip" de poucos centímetros. Desta forma, a fabricação de sistemas analíticos compactos que empregam detecção amperométrica tem sido proposta na literatura e o aspecto diferencial prende-se à possibilidade de realizar todas as operações analíticas de maneira integrada e contínua em um único instrumento ${ }^{8,15,16}$. Há vários exemplos na literatura em que a associação da eletroforese capilar com detecção amperométrica é proposta no desenvolvimento de equipamentos internacionalmente conhecidos como $\mu$ TAS ("micro total analysis system") ou "lab-on-a-chip"18-20. Ainda no que tange ao uso de detectores amperométricos em sistemas hidrodinâmicos, cumpre destacar que a possibilidade de redução da exposição do eletrodo a contaminações provenientes de amostras reais constitui-se em fator relevante e que justifica os seguidos progressos oriundos da introdução de amostras por sistemas FIA ou BIA ("batch injection analysis") $)^{21,22}$.

\section{Sensores eletroquímicos: um panorama resumido}

Sensores químicos são dispositivos que permitem a coleta de dados e obtenção de informações com manipulação mínima do sistema estudado. Desta forma, os resultados obtidos podem ser analisados e correlacionados com outros parâmetros no ambiente em que estão inseridos. Estes dispositivos possuem características peculiares que os distinguem de métodos instrumentais de largo porte, os quais, por usa vez, são cada vez mais precisos, sensíveis e seletivos, mas não permitem a obtenção de informações in situ e em 
tempo real. Dados nestas condições experimentais são facilmente obtidos com sensores e, mesmo que as medidas não tenham precisão e exatidão comparáveis às dos métodos instrumentais, em muitas ocasiões têm-se elementos suficientes para tomadas de decisão. Características vantajosas também inerentes ao uso de sensores químicos referem-se à portabilidade, facilidade de automação, possibilidade de miniaturização e baixo custo.

A Figura 1 apresenta um esquema geral dos principais componentes de um sensor químico. A obtenção de informação analítica depende essencialmente da capacidade da membrana, usualmente posicionada na extremidade do dispositivo, em reconhecer a espécie de interesse de maneira seletiva. Há inúmeras alternativas de imobilização desta membrana na superfície do sensor e um aspecto relevante associado a esta operação envolve a necessidade de acesso a algum processo químico que viabilize a transdução do sinal para o detector. Por sua vez, o sinal transmitido deve ser maximizado em relação às informações sobre o analito, ou seja, deseja-se minimizar o efeito de contribuições estranhas como, por ex., interferentes, ruídos eletrônicos ou erros experimentais. Neste sentido, a efetiva capacidade do dispositivo em discriminar o analito constitui-se em aspecto de grande importância na fabricação de sensores de uso geral e em larga escala, devendo-se ressaltar, entretanto, que aspectos referentes à sensibilidade, estabilidade e robustez também devem ser considerados.

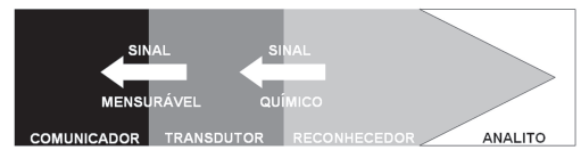

Figura 1. Esquema geral dos principais componentes de um sensor químico

Dentre os sensores químicos, há várias classificações possíveis as quais podem se basear no tamanho, tipo de aplicação ou mecanismo de transdução da resposta. De acordo com o banco de dados do ISI Web of Knowledge ${ }^{\mathrm{SM}}, 46702$ artigos científicos foram publicados contendo a palavra-chave "sensor" ou "sensors", desde 2000. Estes trabalhos referem-se a investigações com sensores de diversos tipos e incluem transdução potenciométrica, amperométrica, piezoelétrica, óptica, térmica, condutométrica, entre outras. Percebe-se, portanto, a vastidão de uma área vigorosamente crescente e não é intenção deste artigo fazer uma revisão de amplitude tão larga. Mesmo no que concerne às técnicas eletroquímicas, verifica-se a existência de milhares de trabalhos sobre o assunto em que são abordados tópicos sobre novos materiais, aplicações em amostras ambientais, biológicas e de interesse industrial, novos métodos de fabricação, estratégias para melhoria na seletividade e nos limites de detecção, etc. $\mathrm{O}$ vigor nesta área é atestado pela publicação de 2678 artigos em igual período de tempo, selecionados com base nas palavras-chave "electrochemical and sensor" e "electrochemical and sensors", alguns dos quais consistindo em excelentes e abrangentes revisões relativamente recentes sobre o tema ${ }^{23-34}$. Aplicações destes dispositivos para obtenção de informações no campo e em amostras de interesse ambiental ${ }^{23,24,30,33}$ exemplificam a importância desta área de pesquisa no contexto social, econômico e da saúde.

A fabricação de sensores descartáveis ${ }^{35-37}$ e o desenvolvimento de tecnologias que permitem a imobilização de enzimas em sistemas eletródicos para confecção de biossensores com elevada estabilidade também têm atraído o contínuo interesse de pesquisadores ligados à Eletroanalítica ${ }^{38-42}$. Sofisticados métodos para "screenprinting" surgem rotineiramente na literatura ${ }^{43-46}$ e a possibilidade de fabricação de microeletrodos isolados ou na forma de "arrays", os quais viabilizam ganho de sensibilidade e abrem perspectivas para monitoramento multielementar ${ }^{47,48}$, impulsionam pesquisas com vistas a aplicações rotineiras dos dispositivos.

\section{O foco do presente artigo}

Como pôde ser evidenciado nos tópicos anteriores, existe uma ampla gama de motivações e inovações tecnológicas que ampliaram de maneira exponencial as fronteiras de atuação da Eletroanalítica e, em particular, o campo associado ao desenvolvimento de sensores eletroquímicos. Como já frisado, há revisões extensas e recentes sobre o tema, incluindo compilações na área de eletrodos modificados e biossensores. De outro lado, a aplicação de sensores amperométricos no estudo de sistemas biológicos constitui-se em área menos explorada, mas que tem recebido incentivo devido às atraentes perspectivas. Em um contexto crescentemente interdisciplinar, em que a busca de informações em sistemas biológicos tem sido cada vez mais importante para compreensão do mecanismo de funcionamento das células, destaca-se a área de fabricação de sensores miniaturizados. Os resultados de experimentos eletroquímicos conduzidos com eletrodos que possuem pelo menos uma das dimensões em escala micrométrica são bastante distintos em função do diferenciado mecanismo de transporte de massa (vide seção adiante), viabilizando medições de corrente em estado estacionário (ou seja, o sinal medido independe do tempo) ${ }^{49-}$ ${ }^{52}$. Outra particularidade destes eletrodos prende-se à possibilidade de construção de dispositivos estruturalmente microscópicos e desta forma, se descortina um vasto campo de atuação da eletroquímica em que se estudam moléculas confinadas em volumes muito pequenos de solução. A presente revisão almeja contextualizar este assunto apresentando alguns dos princípios fundamentais que norteiam os progressos neste campo de pesquisa, assim como exemplos ilustrativos de aplicações práticas destes sensores miniaturizados.

No ambiente celular, os dispositivos usualmente operam no modo amperométrico e, portanto, apresentam reduzida seletividade. Justificam-se, desta maneira, estratégias para a modificação da superfície eletródica, quer seja para fins de eletrocatálise, como para restrição da passagem de espécies interferentes. Comparativamente aos inúmeros trabalhos descritivos sobre métodos de modificação de superfícies eletródicas, menos ênfase tem sido dada na literatura ao uso de ferramentas para a compreensão dos diversos fatores que influenciam a resposta nos eletrodos modificados. Assim, pretende-se abordar o assunto relativo a eletrodos modificados levando em consideração aspectos vinculados a propriedades da estrutura, composição e dinâmica das reações eletródicas e, sempre que possível, exemplos de nossas contribuições nesta área serão apresentados.

\section{ASPECTOS FUNDAMENTAIS SOBRE SENSORES ELETROQUÍMICOS}

\section{Importância da modificação da superfície do eletrodo}

Os sensores amperométricos constituem-se em dispositivos mantidos em potencial fixo e que propiciam sinais de corrente faradaica proporcionais à concentração do analito, em função de processos eletródicos que ocorrem na interface eletrodo/solução. Todavia, a corrente medida em experimentos eletroquímicos também possui um componente capacitivo, associado a mudanças da capacitância da dupla camada elétrica durante variações de potencial. A Figura 2 representa, de maneira esquemática, a influência da concentração da espécie eletroativa para ambas as situações e observa-se claramente que a busca de informações sobre a concentração da espécie eletroativa depende de uma nítida distinção entre as correntes capacitiva e faradaica. Pela análise da figura conclui-se que informações analíticas em concentra- 


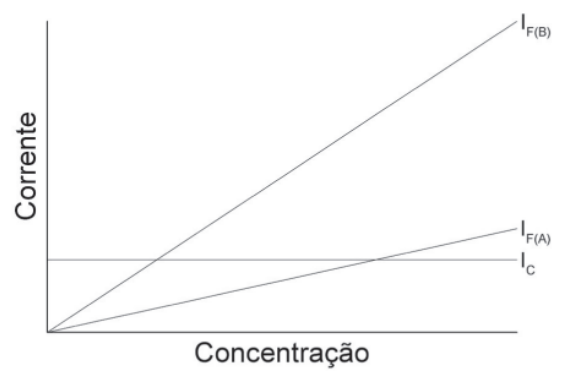

Figura 2. Influência da concentração da espécie eletroativa nas correntes capacitiva $\left(I_{C}\right)$ e faradaica $\left(I_{F}\right.$ em duas situações A e B)

ções mais baixas podem ser obtidas com mais confiabilidade melhorando-se a sensibilidade das determinações (aumento da inclinação da reta, curva $\mathrm{I}_{\mathrm{F}(\mathrm{B})}$ ) e/ou pela diminuiç̧ão da corrente capacitiva, condições nas quais a discriminação entre correntes faradaica e capacitiva é otimizada. Visto que no caso de sensores amperométricos o potencial é mantido constante durante a medição da corrente, deve-se destacar também a importância da avaliação da seletividade das determinações uma vez que, via de regra, em amostras complexas podem existir espécies químicas que também são eletroativas no potencial selecionado.

Uma estratégia elegante para superar os problemas de seletividade acima considerados envolve a modificação da superfície eletródica. Algumas décadas atrás três atributos principais eram requeridos em um eletrodo de trabalho utilizado em experimentos eletroquímicos: boa condutividade, estabilidade química perante processos redox em solução e larga faixa de potencial de trabalho. Desde então, essas premissas passaram a ter um significado menos importante uma vez que, para determinados sistemas químicos, percebeu-se a necessidade do trabalho com superfícies seletivamente reativas, as quais proporcionavam resultados favoráveis em razão da melhoria da atividade química ${ }^{53}$.

$\mathrm{O}$ uso de reagentes moleculares para manipular deliberadamente a superfície de eletrodos tem vasta aplicação em Eletroanalítica, pois conduz a melhoria na capacidade de reconhecimento e/ou na amplificação de sinais de corrente, ao mesmo tempo em que pode tornar as determinações mais seletivas pelo efeito eletrocatalítico (diminuição da sobretensão dos processos eletródicos) ou pela restrição da passagem de espécies interferentes empregando-se membranas apropriadas.

Os procedimentos acima descritos têm estreita associação com o termo "eletrodos quimicamente modificados" ${ }^{\prime 4} \mathrm{e}$ visto que o objetivo do uso de sensores amperométricos está centrado na medição de sinais de corrente em matrizes de natureza complexa (fluidos biológicos, águas naturais, alimentos, etc), a modificação da superfície eletródica é, na maioria dos casos, necessária para que se atinjam os propósitos desejados. Entretanto, cabe destacar que em algumas situações favoráveis as medições podem ser feitas com eletrodos sem qualquer tipo de modificação, como no caso do monitoramento da concentração de ácido ascórbico em frutas cítricas empregando-se microeletrodos de ouro ${ }^{55}$ e platina ${ }^{56}$. Isto é viável pois estudos realizados com suco de laranja por nosso grupo de trabalho empregando a enzima ascorbato oxidase demonstraram a ausência de outras espécies eletroativas no potencial de trabalho. Outro exemplo também interessante é a determinação direta de vitamina $\mathrm{E}$ em óleos e gorduras vegetais utilizando microeletrodos de platina ${ }^{57}$.

A importância da funcionalização da superfície eletródica propiciou a criação de uma área com fronteiras bastante abrangentes e nas últimas décadas os trabalhos têm se estendido desde estudos fundamentais até aplicações práticas. A engenhosidade dos químicos tem levado à proposição de inúmeras estratégias para a imobilização deliberada de espécies químicas na superfície de eletrodos sólidos e há revisões bastante completas sobre este assunto ${ }^{54,58-62}$, razão pela qual não se pretende abordar este tema no presente artigo.
Técnicas para caracterização da atuação do filme modificador

O trabalho com monocamadas é desejável, pois pode-se ter um controle mais preciso do grau de funcionalização da superfície ${ }^{63}$. Todavia, na maior parte dos casos trabalha-se com multicamadas, que viabilizam um efeito catalítico em escala tridimensional devido à difusão do substrato pelo filme ${ }^{64}$. Investigações com filmes mais espessos dão ensejo a uma influência significativa da estrutura da camada imobilizada na dinâmica do processo global de eletrodo e inter-relações entre estes dois parâmetros são bastante relevantes ao se lidar com eletrodos modificados.

$\mathrm{Na}$ avaliação da eficiência catalítica de um eletrodo modificado, fatores relacionados à difusão do substrato na solução, propagação da carga elétrica no filme, penetração do substrato no filme e posterior difusão e velocidade da etapa de transferência eletrônica entre mediador e substrato devem ser considerados ${ }^{65-69}$. A Figura 3 ilustra as diferentes etapas deste processo dinâmico, investigado por Saveant e colaboradores ${ }^{66}$. Estes autores estudaram de maneira qualitativa e quantitativa os perfis de concentração esperados para situações em que os parâmetros acima descritos são considerados. O objetivo destes estudos consiste na avaliação da relevância da contribuição de cada um dos parâmetros na cinética do processo global de eletrodo. As diferentes combinações produzem subcasos definidos pela localização da zona de reação no filme, os quais podem ser caracterizados aumentando-se o regime de transporte de material à superfície eletródica. Para tanto, o uso de eletrodos rotativos é muito conveniente, pois pode-se variar de maneira controlada o coeficiente de transporte de massa variando-se a velocidade de rotação do eletrodo.

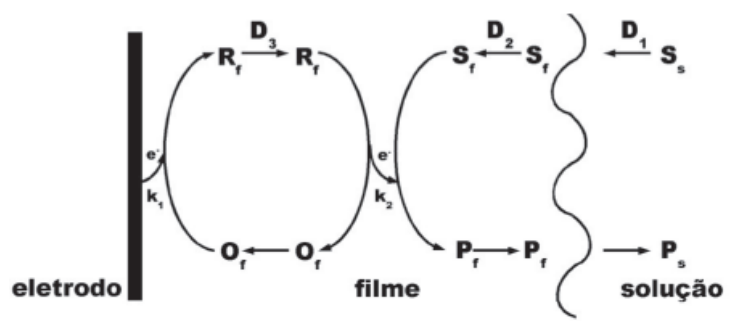

Figura 3. Esquema simplificado das várias etapas relacionadas à redução de um substrato $(S)$ em um eletrodo modificado. Os índices s e f referem-se à difusão do substrato na solução e no filme, respectivamente. D representa o coeficiente de difusão da espécie em solução $\left(D_{1}\right)$ e no filme $\left(D_{2}\right), D_{3}$ referese ao coeficiente de difusão de transporte de carga e $\mathrm{k}_{1}$ e $\mathrm{k}_{2}$ representam as constantes cinéticas dos processos de transferência eletrônica heterogênea e homogênea. Adaptado da ref. 69

Em termos analíticos, onde se deseja maximizar o sinal de corrente e manter uma relação linear com a concentração do substrato, o sistema ideal seria aquele para o qual a reação mediada ocorresse de maneira uniforme no interior do filme ${ }^{68}$. Esta condição é obtida para sistemas químicos em que a reação entre mediador e substrato possui cinética moderada e para os quais não há limitação pelo transporte de carga ou transporte de massa. Deve-se ressaltar, entretanto, que nestes casos o dispositivo pode apresentar restrições para lidar com elevadas taxas de material eletroativo proveniente da solução. Um exemplo típico de sistema com esta característica consiste no processo de oxidação anódica de nitrito em filmes de porfirinas de rutênio, cuja investigação com eletrodo rotativo permitiu a determinação da constante cinética da reação entre nitrito e sítios de $\mathrm{Ru}(\mathrm{III})$ eletrogerados em potenciais suficientemente positivos ${ }^{70}$.

A modificação da superfície eletródica com compostos que facilitam os processos de transferência eletrônica proporciona um 
desafio importante no que diz respeito a investigações sobre composição, estrutura molecular e morfologia. Isto se deve ao fato de que os filmes são depositados em superficies planas e a quantidade de material associada a uma monocamada tem um valor típico de $10^{-10} \mathrm{~mol} / \mathrm{cm}^{2}$, portanto, as técnicas empregadas devem possuir alta sensibilidade. Há artigos de revisão e textos mais aprofundados em que se discutem fundamentos e aplicações de ferramentas espectroscópicas na análise de superficies ${ }^{3,71-74}$. Nosso grupo tem empregado algumas das técnicas acima relacionadas na investigação da estrutura de filmes de óxidos de molibdênio, nos quais se depositaram micropartículas de metais nobres, como platina, paládio e ródio ${ }^{75,76}$. A formação de óxidos e hidróxidos de cobre também foi averiguada empregando-se EDS (“energy dispersive spectroscopy”) e os resultados indicaram a forte relação entre a composição dos filmes e a atividade eletroacatalítica do processo de oxidação do etanol com o potencial aplicado ao eletrodo de trabalho ${ }^{77}$.

Interpretações mais consolidadas sobre o significado químico do evento observado, principalmente quando este envolve variação de massa na interface durante o experimento, podem ser também obtidas empregando-se a balança eletroquímica de cristal de quartzo ${ }^{78,79}$. Esta fornece informações de natureza não-eletroquímica (variação da massa do eletrodo via medição da frequiência de vibração do cristal de quartzo) como, por ex., a cinética de crescimento de filmes ou o mecanismo de transporte iônico, sem perturbar o processo eletroquímico. Além disso, em alguns casos em que a estequiometria do processo eletródico é bem estabelecida, é possível correlacionar em tempo real a massa de filme eletrodepositado na superfície do eletrodo por duas técnicas diferentes, medindo-se a variação na freqüência e a carga. A utilidade desta técnica na investigação de processos eletrocatalíticos foi por nós demonstrada no estudo da oxidação anódica do etanol em eletrodos de cobre em meio alcalino ${ }^{80}$.

\section{Considerações sobre a seletividade das determinações}

Conforme já discutido, uma das grandes limitações das técnicas eletroquímicas com finalidades analíticas tem vínculo com a carência de seletividade das determinações. Se a esta dificuldade se adicionar a limitação do uso contínuo do sensor em função de eventuais problemas com envenenamento, compreende-se o enorme esforço da comunidade eletroquímica na busca de alternativas para restringir o acesso de determinadas espécies químicas à superfície eletródica. Neste sentido, a estratégia convencionalmente utilizada consiste na deposição de membranas poliméricas insolúveis em meio aquoso, que são seletivas à difusão de compostos dissolvidos em solução por diferentes mecanismos. Como exemplos de substâncias convencionalmente utilizadas podem ser citados Nafion ${ }^{81-83}$, polipirrol ${ }^{84}$, polianilina ${ }^{85}$ e acetato de celulose $^{86}$. Cabe ressaltar um interessante uso de membrana de gelatina sólida no recobrimento da superfície eletródica, que traz vantagens adicionais relacionadas à menor influência da convecção nas medições, uma vez que a camada protetora possui espessura relativamente grande. Não se observou, entretanto, diminuição significativa da velocidade de transporte do analito à superfície do eletrodo ${ }^{87}$.

O modo de atuação das membranas protetoras baseia-se essencialmente em restrição por carga e tamanho e os polímeros mais utilizados são acetato de celulose e Nafion. No primeiro caso excluem-se substâncias químicas devido à dimensão das moléculas, uma vez que a porosidade do filme pode ser controlada adequadamente alterando-se o pH da solução e o tempo de hidrólise. Desta forma, somente espécies relativamente pequenas têm acesso à superfície do eletrodo (ou aos sítios catalíticos). No caso do Nafion, o controle da passagem de espécies carregadas é efetuado devido à imobilização de filme contendo sítios aniônicos (sulfonato) dispostos de maneira estruturada no filme. Desta forma, o polímero depositado res- tringe severamente a passagem de ânions, mas é permeável a cátions e espécies eletricamente neutras. Na Figura 4 apresenta-se de maneira esquemática o recobrimento de uma superfície eletródica com vistas ao bloqueio da passagem de espécies químicas.

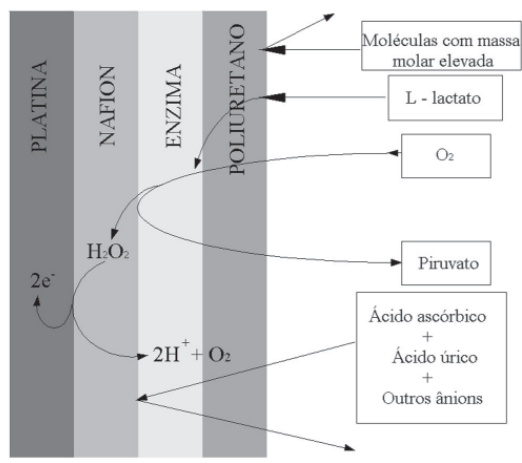

Figura 4. Diagrama esquemático da detecção de L-lactato em microeletrodo de platina. Note a presença de diferentes camadas, que permitem a detecção seletiva de L-lactato. Adaptado da ref. 137

\section{MEDIÇÕES EM AMBIENTES MICROSCÓPICOS}

\section{Miniaturização dos dispositivos}

Seguindo uma tendência da ciência contemporânea, a miniaturização de sistemas eletroquímicos também constitui-se em área de fronteira das pesquisas, embora o trabalho com eletrodos de dimensões micrométricas remonte à década de 40, quando a necessidade de monitorar a concentração de oxigênio em organismos vivos originou a idéia da introdução de eletrodos de platina, de dimensões reduzidas, em tecidos e músculos ${ }^{88}$. Polarizando-se os eletrodos em potenciais suficientemente negativos, foram obtidos sinais de corrente proporcionais à concentração de oxigênio com a vantagem de que as pequenas correntes oriundas do processo faradaico não causavam danos às células.

O termo "microeletrodos" está associado à obtenção de situações de estado estacionário em janelas de tempo muito mais curtas que as usualmente observadas ao se trabalhar com eletrodos de dimensões milimétricas. Há várias maneiras de se obter condições de estado estacionário como, por ex., garantindo a contínua chegada de material eletroativo à superfície do eletrodo por convecção forçada e controlada (eletrodo rotativo ou com células do tipo "wall-jet") ou pela utilização de um segundo eletrodo de trabalho (localizado nas proximidades do eletrodo principal), cuja função é minimizar as perdas oriundas da difusão planar regenerando-se continuamente a espécie eletroativa ("positive feedback effect") ${ }^{89}$. Outra possibilidade envolve o uso de eletrodos com geometria suficientemente apropriada para garantir um fluxo convergente do material eletroativo, de forma a manter um fluxo contínuo em direção ao eletrodo de trabalho. Para tanto, eletrodos que possuem escala micrométrica em pelo menos uma de suas dimensões são bastante eficientes, pois o tempo necessário para o atingimento do estado estacionário (como consequiência da difusão convergente) é tanto menor quanto mais reduzida for esta dimensão. Desta forma, o trabalho com microeletrodos reveste-se de importância na medida em que se minimizam os efeitos da convecção natural, sempre presente alguns segundos após o início dos experimentos.

O desenvolvimento contínuo da eletrônica e da indústria de precisão tem possibilitado a construção de eletrodos com área eletroquimicamente ativa cada vez menor, impulsionando pesquisas envolvendo tais microelectrodos (ou ultramicroeletrodos) na área de estudos eletroquímicos fundamentais ou na aplicação dos mesmos com 
finalidades analíticas. Há inúmeras revisões sobre este assunto, incluindo métodos de fabricação e aplicações ${ }^{49-52,90-92}$. Cabe ressaltar, entretanto, que existem demandas crescentes para o desenvolvimento de métodos analíticos para monitoramento de espécies químicas em micro-ambientes, particularmente no caso de amostras de elevado custo ou nas quais o número de moléculas é muito pequeno. Análises em nano ou até mesmo pico-litros têm sido requeridas e neste contexto situam-se amostras de interesse biológico ${ }^{4,93-97}$. A potencialidade analítica do uso de microeletrodos como sensores eletroquímicos despertou o interesse na construção de dispositivos que, além de possuírem as propriedades eletroquímicas inerentes aos microeletrodos, pudessem ser fabricados de maneira estruturalmente micrométrica, viabilizando o uso em ambientes microscópicos ${ }^{84,95}$. Nestes casos, o isolamento de microfibras condutoras é feito sem o uso de suportes relativamente volumosos, como os utilizados classicamente (micropipetas de vidro, ponteiras de plástico, etc). Tintas eletroforéticas, Teflon e outros materiais têm sido largamente empregados para estes fins e há certamente inúmeras outras alternativas que podem satisfazer as necessidades requeridas em função do tipo de amostra, frequiência de uso e tamanho do dispositivo desejado ${ }^{98-100}$.

Objetivando a fabricação de dispositivos estruturalmente microscópicos, temos estudado procedimentos para corrosão de microfibras de platina e ouro (diâmetro típico de $50 \mu \mathrm{m}$ ) em que, utilizando-se transformadores AC e soluções de trabalho apropriadas, se obtêm extremidades "afinadas", como as mostradas na Figura 5. Por fim, as fibras são seladas pela imersão em um plastificante de borracha e posteriormente deixadas com a ponta para cima até a secagem completa ${ }^{101}$.

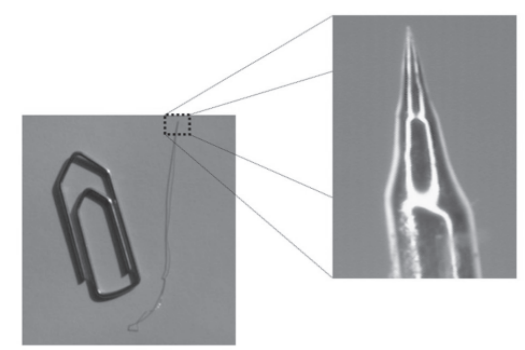

Figura 5. Foto de um microeletrodo de ouro resultante do procedimento de corrosão da fibra utilizando transformadores AC e soluções apropriadas (ver ref. 97 para maiores detalhes)

Finalmente, deve-se destacar a importância da incorporação do eletrodo de referência ao corpo do eletrodo de trabalho, principalmente no caso de sistemas químicos cujo estudo requer a utilização de dispositivos de dimensões muito pequenas. Há diversos procedimentos descritos na literatura ${ }^{84,102}$ com vistas à fabricação de eletrodos com estas características. O mais usual envolve a introdução de uma microfibra dentro do suporte do eletrodo, seguida de deposição de uma fina camada de prata por "sputtering" na camada externa. Esse microeletrodo é, então, mergulhado em solução de $\mathrm{HCl}$ e aplicando-se potencial apropriado o filme de prata é convertido em filme de $\mathrm{AgCl}$ no corpo do capilar ${ }^{84,103,104}$.

\section{Importância e considerações sobre medições em ambientes microscópicos}

A comunicação entre células é efetuada por biomoléculas, como catecolaminas, insulina e dopamina, entre outras, e a habilidade de monitoramento da concentração destes compostos é muito importante em estudos fisiológicos relacionados à transmissão de sinais para o sistema nervoso central. Como já relatado anteriormente, o uso de sensores eletroquímicos de dimensões estruturalmente microscópicas constitui-se em poderosa ferramenta para este tipo de estudo, em que se deseja realizar análises em microambientes e em tempo real. Esta característica essencial dos sensores eletroquímicos os distinguem com vantagens comparando-se com procedimentos bem estabelecidos na literatura em que são empregadas técnicas de extração de amostras em regiões adjacentes à célula. Neste caso, volumes muito pequenos (da ordem de $\mathrm{nL}$ ou mesmo $\mathrm{pL}$ ) são removidos do ambiente celular com auxílio de micropipetas para posterior análise por técnicas analíticas convencionais, como as espectroscópicas ou de separação, e especial atenção tem sido dada à eletroforese capilar. Ganhase em seletividade, pois as técnicas citadas podem conduzir a informações sobre a composição da amostra, mas perde-se em resolução temporal e espacial ${ }^{105}$. Uma excelente revisão sobre "single-cell analysis" foi publicada recentemente e nela podem ser encontradas informações detalhadas sobre as metodologias usualmente empregadas neste tipo de investigação ${ }^{106}$. Avaliações críticas sobre as vantagens e desvantagens da análise de células individuais têm sido apresentadas na literatura ${ }^{107}$ e como aspectos positivos considera-se a importância da localização das moléculas na célula e a análise de comportamentos individuais que se diluem em populações. De outro lado, problemas vinculados à representatividade dos resultados têm sido apontados, assim como o fato de que informações diferentes podem ser obtidas não como resultado do comportamento da célula, mas sim dos diferentes estágios do processo de divisão.

A possibilidade de mapear o microambiente vizinho à célula constitui-se na grande vantagem dos sensores amperométricos neste tipo de estudo ${ }^{108}$. Uma das características necessárias do sensor empregado é a elevada resolução espacial em escala tridimesional e, para tanto, a fabricação de dispositivos com geometria microscópica é essencial. O tempo de resposta do dispositivo deve ser muito baixo, para que os dados sobre os eventos possam ser adquiridos em tempo real, e tanto a seletividade como a sensibilidade das determinações devem ser suficientemente altas, para que os sinais medidos tenham perfeita correlação com as informações desejadas.

Um aspecto a ser considerado no uso de sensores eletroquímicos para obtenção de informações sobre a liberação de biomoléculas em ambientes celulares diz respeito à eventual distribuição nãouniforme da espécie química de interesse no espaço amostrado. Diferentemente de medições realizadas em meio homogêneo, nestes casos cria-se um gradiente de concentração, de forma que o significado da medição efetuada em um ponto muito específico do ambiente celular deve ser analisado com cuidado. Este efeito é tanto mais pronunciado quanto menor for a distância entre a fonte emissora da biomolécula e a extremidade do sensor, razão pela qual a resposta do dispositivo deve ser muito rápida para monitorar com resolução as variações na composição do microambiente.

Outro detalhe a ser considerado durante medições em amostras de dimensões reduzidas tem relação com a perturbação no sistema ocasionada pela eletrólise. Nestas situações, em que o número de moléculas é extremamente pequeno, as modificações na composição da solução podem ser significativas, tanto pela remoção do analito estudado, como também pela introdução de novas espécies químicas resultantes do processo eletródico. Estudos têm sido realizados com intuito de avaliar a extensão da eletrólise nestes microambientes, tipicamente da ordem de alguns pL, e de fazer comparações com processos similares conduzidos em condições convencionais (volumes não restritos). As perdas por evaporação são minimizadas pelo recobrimento da solução aquosa com óleos minerais ou acondicionamento da amostra em "microvials" ou reservatórios especialmente fabricados por litografia ${ }^{93-95,97}$. Estas estratégias são bastante úteis quando se deseja monitorar a concentração total da espécie liberada durante um evento no ambiente celular e, visto que as moléculas 
podem ser mantidas em volumes muito reduzidos, pode-se trabalhar com concentrações relativamente altas.

\section{Determinações amperométricas in vivo de espécies químicas de importância biológica}

Conforme já frisado em seção anterior, o emprego de sensores eletroquímicos para monitoramento da concentração de moléculas de relevância biológica tem sido objeto de investigações crescentes e profícuas em Eletroanalítica, motivadas especialmente pela versatilidade dos procedimentos associados à técnica. Uma das espécies mais estudadas consiste no óxido nítrico (NO), molécula reconhecida como mensageiro celular no corpo humano. O papel do NO na evolução de diversas doenças relacionadas ao coração e à impotência sexual tem motivado estudos sobre comportamento bioquímico e propriedades terapêuticas. A rápida difusão da molécula do centro gerador ao sítio de atuação e a elevada reatividade química justificam o desenvolvimento de dispositivos confiáveis, precisos, sensíveis e de resposta rápida para viabilizar a compreensão dos mecanismos de atuação do NO.

O desenvolvimento de sensores eletroquímicos para medições de NO in vivo remonta ao início da década de 90, quando foram publicados os primeiros trabalhos que nortearam o princípio de fabricação de sensores comercias para NO. Estes baseiam-se no recobrimento de microeletrodos de platina com filmes eletropolimerizados de porfirinas de níquel ${ }^{109,110}$. O papel destes filmes envolve processo eletrocatalítico de oxidação do NO e na presença de membranas de Nafion restringe-se a oxidação de espécies aniônicas presentes nos fluidos biológicos como, por ex., ascorbato. Algumas revisões sobre o assunto podem ser encontradas na literatura $^{111-115}$, assim como exemplos de recentes aplicações destes sensores eletroquímicos para monitoramento de NO em amostras biológicas ${ }^{114,116-119}$. Nossas contribuições nesta área envolveram estudos sobre a codeposição de complexos de rutênio e óxidos de molibdênio em microeletrodos de ouro e pôde-se constatar a viabilidade do uso do dispositivo no monitoramento da variação da concentração de NO liberado em reação química envolvendo peroxonitrito e tempol em meio de bicarbonato ${ }^{120}$.

Pode-se constatar a importância do uso de microeletrodos como sensores para neurotransmissores ${ }^{121}$ em nível celular pelos inúmeros trabalhados publicados recentemente na literatura. Compostos como glutamato ${ }^{122}$, catecolaminas ${ }^{123,124}$, dopamina ${ }^{125-127}$, serotonina $^{124,127}$, entre outros, têm sido determinados com seletividade, sensibilidade e tempo de resposta adequados, fornecendo subsídios para melhor compreensão dos processos de transmissão de informações entre células nervosas.

O glutamato é o principal neurotransmissor excitatório do cérebro de mamíferos e em altas quantidades pode causar desordens neurológicas, incluindo esquizofrenia, doença de Parkinson, derrame e epilepsia. Portanto, sensores capazes de monitorar a liberação desta espécie química em tempo real podem contribuir para melhor entendimento dos estados fisiológico e patológico induzidos pelo glutamato no sistema nervoso central. Quanto à determinação de glutamato in vivo, há uma grande quantidade de trabalhos recentes relatados na literatura ${ }^{122,128-130}$.

Algumas purinas, tais como ATP e adenosina, também são de grande importância biológica por serem indicativos de distúrbios neurológicos. Por esta razão, um aumento crescente no número de artigos publicados na literatura envolvendo a fabricação de microeletrodos para determinação destas purinas em tempo real tem sido evidenciado ${ }^{131-133}$.

Outros compostos gerados como respostas a estímulos às células também têm sido determinados com sensores eletroquímicos e, dentre eles, podem-se citar peróxido de hidrogênio ${ }^{134}$, lactato $^{135-137}$ e superóxidos ${ }^{138,139}$. A possibilidade de monitoramento de mais de um composto na superfície do cérebro com um dispositivo único constituído por conjunto de microeletrodos de disco separados foi discutida por Michael e colaboradores ${ }^{125}$. A seletividade das respostas obtidas para glutamato, glicose e colina foi demonstrada em trabalho em que enzimas são suportadas na superfície de microletrodos de fibra de carbono com auxílio de filmes poliméricos ${ }^{123}$. Propostas para determinação simultânea de catecolaminas e serotonina ${ }^{124}$ ou serotonina e dopamina ${ }^{127}$ também são encontradas na literatura.

O lactato é conhecido como um dos mais importantes metabólitos em análise clínica e consiste em indicador de choques, insuficiência respiratória, doenças cardíacas e desordem metabólica. Os níveis de lactato podem também ser associados a problemas nos tecidos, à trombose e às condições físicas de animais e atletas. Por este motivo, novas tecnologias para dosagem do lactato vêm sendo estudadas em conjunto com testes laboratoriais realizados fora do ambiente clínico convencional, testes estes chamados de "point-ofcare testing"140. Viabiliza-se, desta maneira, a dosagem da concentração sanguínea do lactato em campo, de forma precisa e com uma pequena gota de sangue obtida da ponta de um dos dedos do atleta. Como o resultado é obtido em poucos minutos, a realização do teste, a interpretação dos resultados e a tomada de decisões são facilitadas, ampliando o uso do indicador na Medicina Esportiva.

\section{PERSPECTIVAS E DESAFIOS}

A viabilidade tecnológica da fabricação de microeletrodos com extremidades muito finas, com dimensões sub-micrométricas, tem alargado o uso da técnica SECM ("scanning electrochemical microscopy") no estudo de sistemas celulares ${ }^{141}$. A elevada resolução espacial da técnica oriunda da dimensão reduzida do eletrodo, associada à rapidez das respostas obtidas em condições de estado estacionário, permite a obtenção de informações sobre a concentração em uma escala tridimensional. Obtêm-se, portanto, mapas topográficos do sistema químico. A técnica também possibilita a geração eletroquímica de espécies químicas em regiões muito específicas, dando ensejo a experimentos sobre a reatividade de moléculas em localidades definidas da célula. Finalmente, cabe ressaltar a importância de se operar no modo "positive feedback" $"{ }^{99,142}$, uma vez que se pode encapsular um pequeno número de moléculas em um ambiente muito restrito. Como a espécie eletroativa pode ser continuamente regenerada no microeletrodo, localizado muito próximo do substrato, mesmo que o número de moléculas seja pequeno podem-se obter correntes mensuráveis ${ }^{142,143}$.

Embora existam vantagens indiscutíveis no uso de sensores eletroquímicos, alguns aspectos ainda estão no limiar de soluções plausíveis quando se pensa em termos "realmente práticos". Um dos desafios para os quais já existem pesquisas em andamento refere-se, por ex., à implantação de sensores no corpo humano ${ }^{144}$. Esta possibilidade é altamente atraente pois eliminam-se as desvantagens dos procedimentos invasivos muito freqüentes que, mesmo para volumes pequenos de amostra, causam inconvenientes. O exemplo mais próximo da vida cotidiana envolve medições do nível de glicose no sangue, para as já quais existem sensores amperométricos comerciais ${ }^{145}$. Entretanto, quando implantados no tecido humano, é de se esperar uma pronta resposta do organismo contra a "invasão", resultando no desenvolvimento de camadas protetoras que tendem a isolar o dispositivo do ambiente celular. Outros ítens a serem considerados relacionam-se à necessidade de recarregamento da bateria do sensor, à eventual recalibração periódica e à biocompatibilidade dos materiais empregados. Trata-se, portanto, de aspectos de ordem prática que vão requerer investigações aprofundadas para consecução das metas desejadas, ou seja, dispositivos operando a baixo custo e com autonomia suficientemente prolongada para garantir o longo uso. 
Ressalte-se ainda que sistemas miniaturizados de monitoramento constituem-se em ferramenta para coleta e transmissão de dados sobre a saúde de pacientes em ambientes domiciliares ou no trabalho, facilitando a predição de doenças e o possível diagnóstico. Como consequiência, consultas face a face podem ser reduzidas, assim como as estadas em hospitais. Essa tecnologia, denominada telemetria, também pode contribuir para otimização dos recursos destinados à prevenção de doenças, principalmente em ambientes rurais. Trabalhos envolvendo o uso de sistemas telemétricos implantados para monitoramento de atividades neurais ${ }^{146}$, nível de oxigênio no sangue e frequiências cardíaca e respiratória ${ }^{147}$ e arritmias $^{148}$ já estão relatados na literatura.

Em resumo, o futuro da área de pesquisas sobre sensores eletroquímicos, especialmente aqueles destinados ao uso com fins de monitoramento em sistema biológicos, certamente vai envolver o conceito de interdisciplinaridade. A preparação de novos polímeros e matrizes hospedeiras aos quais se alojam catalisadores eficientes consiste em área onde químicos de diferentes especialidades podem atuar e, neste sentido, são relevantes não só pesquisas aplicadas mas também aquelas de cunho fundamental. A imobilização do material em eletrodos de dimensões nano ou micrométricas necessita de estudos contínuos na área de novos materiais e nanotecnologia. Investigações e novos desenvolvimentos relacionados ao uso do sensor eletroquímico no monitoramento de metabólitos de reações enzimáticas ou substâncias de interesse bioquímico podem ser de valia tanto para profissionais ligados à área de Química, como também para aqueles ligados às áreas biológicas. A construção de dispositivos portáteis e a recepção e transmissão fidedigna de dados a longas distâncias (telemetria) vai depender da interação com engenheiros. Conclui-se, portanto, que a linha de pesquisa envolvendo sensores eletroquímicos vai suplantar em um futuro próximo as fronteiras da academia e atrair a atenção das áreas tecnológicas e comerciais, demonstrando a importância deste ramo da ciência para a melhoria das condições de vida em nossa sociedade.

\section{AGRADECIMENTOS}

À FAPESP, CAPES e ao CNPq pelo apoio financeiro concedido na forma de bolsas e para o financiamento das pesquisas.

\section{REFERÊNCIAS}

1. Brett, C. M. A.; Brett, A. M. O.; Electroanalysis, Oxford University Press; Oxford, 1998.

2. Wang, J.; Analytical Electrochemistry, $2^{\text {nd }}$ ed., John Wiley \& Sons, Inc.: New York, 2000.

3. Bard, A. J.; Faulkner, L. R.; Electrochemical methods: Fundamentals and Applications, John Wiley \& Sons, Inc.: New York, 2001.

4. Brattern, C. D. T.; Cobbold, P. H.; Cooper, J. M.; Anal. Chem. 1997, 69, 253.

5. Tur'yan, Y. I.; Talanta 1997, 44, 1.

6. Brett, C. M. A.; Electroanalysis 1999, 11, 1013.

7. Wang, J.; Tian, B.; Wang, J.; Lu, J.; Olsen, C.; Yarnitzky, C.; Olsen, K.; Hammestrom, D.; Bennett, W.; Anal. Chim. Acta 1999, 385, 429.

8. Štulík, K.; Electroanalysis 1999, 11, 1001.

9. Cai, X.; Glidle, A.; Cooper, J. M.; Electroanalysis 2000, 12, 631.

10. Suzuki H.; Electroanalysis 2000, 12, 703

11. Feeney, R.; Kounaves, S. P.; Electroanalysis 2000, 12, 677.

12. He, H. X.; Li, Q. G.; Zhou, Z. Y.; Zhang, H.; Li, S. F. Y.; Liu, Z. F.; Langmuir 2000, 16, 9683 .

13. Wang, J.; Talanta 2002, 56, 223.

14. Matysik, F.-M.; Anal. Bioanal. Chem. 2003, 375, 33.

15. Hierlemann, A.; Brand, O.; Hagleitner, C.; Baltes, H.; Proceedings of the IEEE 2003, 91, 839.

16. Ahn, C. H.; Choi, J.-W.; Beaucage, G.; Nevin, J. H.; Lee, J.-B., Puntambekar, A.; Lee, J. Y.; Proceedings of the IEEE 2004, 92, 154.

17. Siuzdak, G.; Mass Spectrometry for Biotechnology, Academic Press: San Diego, 1996
18. da Silva, J. A. F.; Quim. Nova 2003, 26, 56.

19. Yan, J. L.; Du, Y.; Liu, J. F.; Cao, W. D.; Sun, S. H.; Zhou, W. H.; Yang, X. R.; Wang, E. K.; Anal. Chem. 2003, 75, 5406.

20. Richter, E. M.; da Silva, J. A. F.; Gutz, I. G. R.; Angnes, L.; Electrophoresis 2004, 25, 2965.

21. Ruzicka, J.; Hansen, E. H.; Flow Injection Analysis, $2^{\text {nd }}$ ed., John Wiley \& Sons: New York, 1988.

22. Quintino, M. S. M.; Angnes, L.; Electroanalysis 2004, 16, 513.

23. Fleet, B.; Gunasingham, H.; Talanta 1992, 39, 1449.

24. Brett, C. M. A.; Pure Appl. Chem. 2001, 73, 1969.

25. Bakker, E.; Telting-Diaz, M.; Anal. Chem. 2002, 74, 2781.

26. Janata, J.; Crit. Rev. Anal. Chem. 2002, 32, 109.

27. Wang, J.; Acc. Chem. Res. 2002, 35, 811 .

28. Piletsky, S. A.; Turner, A. P. F.; Electroanalysis 2002, 14, 317.

29. Stradiotto, N. R.; Yamanaka, H.; Zanoni, M. V. B.; J. Braz. Chem. Soc. 2003, 14, 159

30. Ashley, K.; J. Hazard Mater. 2003, 102, 1

31. Stetter, J. R.; Penrose, W. R.; Yao, S.; J. Electrochem. Soc. 2003, 150, S11

32. Bakker, E.; Anal. Chem. 2004, 76, 3285.

33. Hanraham, G.; Patil, D. G.; Wang, J.; J. Environ. Monit. 2004, 6, 657.

34. Wróblewski, W.; Dybko, A.; Malinowska, E.; Brzózka, Z.; Talanta 2004, 63, 33.

35. Angnes, L.; Richter, E. M.; Augelli, M. A.; Kume, G. H.; Anal. Chem. 2000, $72,5503$.

36. Cheng, J.; Jandik, P.; Avdalovic, N.; J. Chromatogr., A 2003, 997, 73.

37. Lowinsohn, D.; Richter, E. M.; Angnes, L.; Bertotti, M.; Electroanalysis, no prelo.

38. Bartlett, P. N. Em Biosensor Technology: Fundamentals and Applications; Buck, R. P.; Hatfield, W. E.; Umaña, M.; Bowden, E. F., eds.; Marcel Dekker, Inc.: New York, 1990, cap. 7, p. 95.

39. Alvarez-Icaza, M.; Bilitewski, U.; Anal Chem. 1993, 65, 525A

40. O'Connell, P. J.; Guilbault, G. G.; Anal. Lett. 2001, 34, 1063.

41. Avramescu, A.; Andreescu, S.; Noguer, T.; Bala, C.; Andreescu, D.; Marty, J.-L.; Anal. Bioanal. Chem. 2002, 374, 25.

42. Freire, R. S.; Pessoa, C. A.; Kubota, L. T.; Quim. Nova 2003, 26, 381.

43. Hart, J. P.; Wring, S. A.; Trends Anal. Chem. 1997, 16, 89.

44. Nascimento, V. B.; Angnes, L.; Ouim. Nova 1998, 21, 614

45. Honeychurch, K. C.; Hart, J. P.; Trends Anal. Chem. 2003, 22, 456.

46. Hart, J. P.; Crew, A.; Crouch, E.; Honeychurch, K. C.; Pemberton, R. M.; Anal. Lett. 2004, 37, 789.

47. Herdan, J.; Feeney, R.; Kounaves, S. P.; Flannery, A. F.; Storment, C. W.; Kovacs, G. T. A.; Darling, R. B.; Environ. Sci. Technol. 1998, 32, 131.

48. Williams, G.; D'Silva, C.; Analyst 1994, 119, 2337.

49. Fleischmannn, M.; Pons, S.; Rolison, D. R.; Schmidt; Ultramicroelectrodes, Datatech Systems, Inc.: Morgantown, NC, 1987.

50. Wightman, R. M.; Wipf, D. O. Em Electroanalytical Chemistry: A series of advances; Bard, A. J., ed.; Marcel Dekker, Inc.: New York, 1989, vol. 15 , p. 267.

51. Montenegro, M. I.; Queirós, A. M.; Daschbach, J.; Microelectrodes: theory and applications, NATO ASI series, Riedel: Dordrecht, 1991.

52. Correia, A. N.; Mascaro, L. H.; Machado, S. A. S.; Mazo, L. H.; Avaca, L. A.; Quim. Nova 1995, 18, 475.

53. Murray, R. W.; Acc. Chem. Rev. 1980, 13, 135.

54. Murray, R. W. Em Electroanalytical Chemistry: A series of advances; Bard, A. J., ed.; Marcel Dekker, Inc.: New York, 1984, vol. 13, p. 191.

55. Farrington, A. M.; Jagota, N.; Slater, J. M.; Analyst 1994, 119, 233

56. Paixão, T. R. L. C.; Lowinsohn, D.; Bertotti, M.; J. Agric. Food Chem. 2006, 54, 3072.

57. Coatanea M.; Darchen, A.; Hauchard, D.; Sens. Actuactors, B 2001, 76, 539 .

58. Wring, S. A.; Hart, J. P.; Analyst 1992, 117, 1215.

59. Walcarius, A.; Electroanalysis 1998, 10, 1217.

60. Pereira, A. C.; Santos A. de S.; Kubota, L. T.; Quim. Nova 2002, 25, 1012.

61. Zen, J.-M.; Kumar, A. S.; Tsai, D.-M.; Electroanalysis 2003, 15, 1073.

62. Crespilho, F. N.; Rezende, M. O. O.; Quim. Nova 2004, 27, 964.

63. Murray, R. W.; Electroanal. Chem. 1989, 15, 267.

64. Faulkner, L. R.; Electrochim. Acta. 1989, 34, 1699

65. Laviron, E.; J. Electroanal. Chem. 1982, 131, 61.

66. Andrieux, C. P.; D.-Bouchiat, J. M.; Savéant, J. M.; J. Electroanal. Chem. 1982, 131, 1 .

67. Andrieux, C. P.; D.-Bouchiat, J. M.; Savéant, J. M.; J. Electroanal. Chem. 1984, $169,9$.

68. Albery, W. J.; Hillman, A. R.; J. Electroanal. Chem. 1984, 170, 27.

69. Durst, R. A.; Baumner, A. J., Murray, R. W.; Buck, R. P.; Andrieux, C. P.; Pure Appl. Chem. 1997, 69, 1317.

70. da Rocha, J. R. C.; Demets, G. J. F.; Bertotti, M.; Araki, K.; Toma, H. E.; J. Electroanal. Chem. 2002, 526, 69 . 
71. Wang, J.; Electroanalysis 1991, 3, 255.

72. Christensen, P. A.; Hamnett, A.; Techniques and mechanisms in electrochemistry, $1^{\text {st }}$ ed., Blackie Academic \& Professional: Glasgow, 1994

73. Strausser, Y. E.; Heaton, M. G.; American Laboratory 1994, 26, 20.

74. Duong, B.; Arechabaleta, R.; Tao, N. J.; J. Electroanal. Chem. 1998, 447, 63.

75. Kosminsky, L.; Matos, R. C.; Tabacniks, M. H.; Bertotti, M.; Electroanalysis 2003, 15, 733 .

76. Pereira, A. C.; Ferreira, T. L.; Kosminsky, L.; Matos, R. C.; Bertotti, M. Tabacniks, M. H.; Kiyohara, P. K.; Fantini, M. C. A.; Chem. Mater. 2004, $16,2662$.

77. Paixão, T. R. L. C.; Bertotti, M.; J. Electroanal. Chem. 2004, 571, 101.

78. Buttry, D. A. Em Electroanalytical Chemistry: A series of advances; Bard, A. J., ed.; Marcel Dekker, Inc.: New York, 1991, vol. 17, p. 1.

79. Varela, H.; Malta, M.; Torresi, R. M.; Quim. Nova 2000, 23, 664.

80. Paixão, T. R. L. C.; Ponzio, E. A.; Torresi, R. M.; Bertotti, M.; J. Braz Chem. Soc. 2006, 17, 374.

81. Navy, G.; Gerhardt G. A.; Oke, A. F.; Rice, M. E.; Adams, R. N.; Moore, R. B., Szentirmay, M. N.; Martin, C. R.; J. Electroanal. Chem. 1985, 188, 85.

82. Fungaro, D. A.; Brett, C. M. A.; Quim. Nova 2000, 23, 805.

83. Brown, F. O.; Lowry, J. P.; Analyst 2003, 128, 700.

84. Zhang, X.; Ogorevc, B.; Tavcar, G.; Švegl, I. G.; Analyst 1996, 121, 1817.

85. Wang, Y.; Huang, J.; Zhang, C.; Wei, Zhou, X.; Electroanalysis 1998, 10 , 776.

86. Wilson, G. S.; Sittampalam, G.; Anal. Chem. 1983, 55, 1608

87. Howell, J. O.; Kuhr, W. G.; Ensman, R. E.; Wightman, R. M.; J. Electroanal Chem. 1986, 209, 77

88. Davies, P. W.; Brink, F.; Rev. Sci. Instrum. 1942, 13, 524.

89. Bard, A.J.; Denuault, G.; Lee, C.; Mandler D.; Wipf, D.O.; Acc. Chem. Res. 1990, 23, 357.

90. Silva, S. M.; Alves, C. R.; Correia, A. N.; Martins, R. M.; Nobre, A. L. R.; Machado, S. A. S.; Mazo, L. H.; Avaca, L. A.; Quim. Nova 1998, 21, 78.

91. Štulík, K.; Amatore, C.; Holub, K.; Marecek, V.; Kutner, W.; Pure Appl. Chem. 2000, 72, 1483.

92. Lowinsohn, D.; Peres, H. E. M.; Kosminsky, L.; Paixão, T. R. L. C.; Ferreira T. L.; Ramirez-Fernandez, F. J.; Bertotti, M.; Sens. Actuators, B, no prelo.

93. Clark, R. A.; Hietpas, P. B.; Ewing, A. G.; Anal. Chem. 1997, 69, 259.

94. Clark, R. A.; Ewing, A. G.; Anal. Chem. 1998, 70, 1119

95. Kashyap, R.; Gratzl, M.; Anal. Chem. 1998, 70, 1468.

96. Niwa, O.; Kurita, R.; Liu, Z., Horiuchi, T.; Torimitsu, K.; Anal. Chem. 2000, $72,949$.

97. Troyer, K. P.; Whightman, R. M.; Anal. Chem. 2002, 74, 5370.

98. Schulte, A.; Chow, R. H.; Anal. Chem. 1996, 68, 3054.

99. Zhang, X.; Ogorevc, B.; Anal. Chem. 1998, 70, 1646.

100. Slevin, C. J.; Gray, N. J.; Macpherson, J. V.; Webb, M. A.; Unwin, P. R.; Elechochem. Communn. 1999, 1, 282.

101. Paixão, T. R. L. C.; Jesus, D. P.; Bertotti, M.; Resumos da $28^{a}$. Reunião da Sociedade Brasileira de Química, Poços de Caldas, Brasil, 2005.

102. Turcu, F.; Schulte, A.; Schuhmann, W.; Anal. Bioanal. Chem. 2004, $380,736$.

103. Zhang, X.; Wang, J.; Ogorevc, B.; Spichiger, U. E.; Electroanalysis 1999, 11,945 .

104. Zhang, X.; Lin, J.; Cardosa, L.; Broderick, M.; Fein, H.; Lin, J.; Electroanalysis 2000, 12, 1113

105. Stuart, J. N.; Sweedler, J. V.; Anal. Bioanal. Chem. 2003, 375, 28.

106. Lu, X.; Huang, W.-H.; Wang, Z.-L.; Cheng, J.-K.; Anal. Chim. Acta 2004 $510,127$.

107. Cottingham, K.; Anal. Chem. 2004, 76, 235A.

108. Wightman, R. M.; Runnels, P.; Troyer, K.; Anal. Chim. Acta 1999, 400, 5.

109. Malinski, T.; Taha, Z.; Nature 1993, 358, 676.

110. Malinski, T.; Taha, Z.; Grunfeld, S.; Burewicz, A.; Tomboulian, P.; Anal. Chim. Acta 1993, 279, 135.
111. Malinski, T.; Czuchajowski, L. Em Methods in Nitric Oxide Research; Feelisch, M.; Stamler, J. S., eds.; John Wiley \& Sons Ltd: New York, 1996, cap. 22 , p. 319

112. Pallini, M.; Curulli, A.; Amine, A.; Palleschi, G.; Electroanalysis 1998, 10, 1010 .

113. Allen, B. W.; Piantadosi, C. A.; Coury, L. A., NITRIC OXIDE: Biology and Chemistry 2000, 4, 75.

114. Bedioui, F.; Villeneuve, N.; Electroanalysis 2003, 15, 5.

115. Ciszewski, A.; Milczarek, G.; Talanta 2003, 61, 11

116. Cserey, A.; Gratzl, M.; Anal. Chem. 2001, 73, 3965.

117. Katrlík, J.; Zálešáková, P.; Biochem. 2002, 56, 73.

118. Zhang, X.; Lin, J.; Cardosa, L.; Broderick, M.; Darley-Usmar, V.; Electroanalysis 2002, 14, 697

119. Dickson, A.; Lin, J.; Sun, J. Broderick, M.; Fein, H.; Zhang, X.; Electroanalysis 2004, 16, 640.

120. Mori, V.; Toledo, J. C.; Silva, H. A. S.; Franco, D.W.; Bertotti, M.; J. Electroanal. Chem. 2003, 547, 9.

121. Dale, N.; Hatz, S.; Tian, F.; Llaudet, E.; Trends Biotechnol. 2005, 23, 420.

122. Burmeister, J. J.; Gerhardt, G. A.; Trends Anal. Chem. 2003, 22, 498.

123. Cahill, P. S.; Walker, Q. D.; Finnegan, J. M.; Mickelson, G. E.; Travis, E. R.; Wightman, R. M.; Anal. Chem. 1996, 68, 3180.

124. Pennington, J. M.; Millar, J. Jones, C. P.; Owesson, C. Å; McLaughlin, D. P.; Stamford, J. A.; J. Neurosci. Methods 2004, 140, 5.

125. Cui, J.; Kulagina, N. V.; Michael, A. C.; J. Neurosci. Methods 2001, 104, 183.

126. Dressman, S. F.; Peters, J. L.; Michael, A. C.; J. Neurosci. Methods 2002, $119,75$.

127. Jiang, X.; Lin, X.; Anal. Chim. Acta 2005, 537, 145.

128. Burmeister, J. J.; Pomerleau, F.; Palmer, M.; Day, B. K.; Huettl, P.; Gerhardt, G. A.; J. Neurosci. Methods 2002, 119, 163

129. Oldenziel, W. H.; Beukema, W.; Westerink, B. H. C.; J. Neurosci. Methods 2004, 140, 117.

130. Rahman, M. A.; Kwon, N.-H.; Won, M.-S.; Choe, E. S.; Shim, Y.-B.; Anal. Chem. 2005, 77, 4854

131. Frenguelli, B. G.; Llaudet, E.; Dale, N.; J. Neurochem. 2003, 86, 1506.

132. Llaudet, E.; Botting, N. P.; Crayston, J. A.; Dale, N.; Biosens. Bioelectron. 2003, 18, 43

133. Llaudet, E.; Hatz, S.; Droniou, M.; Dale, N.; Anal Chem. 2005, 77, 3267.

134. Kulagina, N. V.; Michael, A. C.; Anal. Chem. 2003, 75, 4875.

135. Shram, N. F.; Netchiporouk, L. I.; Martelet, C.; Jaffrezic-Renault, N.; Bonnet, C.; Cespuglio, R.; Anal. Chem. 1998, 70, 2618.

136. Wu, M.; Lin, Z.; Li, Y.; Ren, S.; Sens. Actuators, B 2000, 66, 269.

137. Burmeister, J. J.; Palmer, M.; Gerhardt, G. A.; Biosens. Bioelectron. 2005, $20,1772$.

138. Beissenhirtz, M. K.; Scheller, F. W.; Lisdat, F.; Anal. Chem. 2004, 76, 4665.

139. Arbault, S.; Sojic, N.; Bruce, D.; Amatore, C.; Sarasin, A.; Vuillaume, M.; Carcinogenesis 2004, 25, 509.

140. Tüdós, A. J.; Besselink, G. A. J.; Schasfoort, B. M.; Lab on a Chip 2001, 1,83 .

141. Yasukawa, T.; Kaya, T.; Matsue, T.; Electroanalysis 2000, 12, 653.

142. Paixão, T. R. L. C.; Richter, E. M.; Brito-Neto, J. G. A.; Bertotti, M.; Electrochem. Commun., no prelo.

143. Fan, F.-R. F.; Kwak, J.; Bard, A. J.; J. Am. Chem. Soc. 1996, 118, 9669

144. Henry, C.; Anal. Chem. 1998, 70, 594A.

145. Heller, A.; Annu. Rev. Biomed. Eng. 1999, 1, 153.

146. A. Mohseni, P.; Najafi, K.; Eliades, S. J.; Wang, X.; IEEE Trans. Neural Sys. Rehab. Eng. 2005, 13, 263.

147. Tura, A.; Badanai, M.; Longo, D.; Quarenti, L.; J. Telemed. Telecare 2004, $10,298$.

148. http://www.cardguard.com/site/index.asp, acessada em outubro 2005. 